\title{
Special Issue on Multi-Scale Experimental Measurements in Mechanics
}

\author{
Y. Kang • H. Xie • Y.J. Chao
}

Published online: 7 January 2014

(C) Society for Experimental Mechanics 2014

The development of micro/nano science and technology in recent years has created new challenges to the traditional macro-scale based experimental measurement. As such, multi-scale experimental measurements in mechanics have become a key area of research. Both the metrology and its applications have drawn great interest from researchers in related fields and have been progressing rapidly.

It is our pleasure to present the nine papers in this special issue, entitled "Multi-Scale Experimental Measurements in Mechanics." The special issue attempts to gather papers in the developments of multi-scale metrologies and related applications. It covers a range of topics to reflect the recent advances in optical measurement methods, non-destructive techniques, mechanical behavior characterization under different high resolution microscopes, fabrication techniques on micro-deformation sensor, etc. A brief introduction of these papers is as follows:

In regard to multi-scale experiments including uniaxial tensile testing, in situ Raman spectroscopy and scanning electron microscopy, Wei-Lin Deng et al. investigated the deformation in multi-scale and interfacial mechanical behavior of carbon nanotube fibers with multi-level structures. A two-

\section{Y. Kang}

School of Mechanical Engineering, Tianjin University,

Tianjin, China

H. Xie

AML, Department of Engineering Mechanics, Tsinghua University, Beijing, China

Y.J. Chao $(\bowtie)$

Department of Mechanical Engineering, University of South

Carolina, Columbia, SC, USA

e-mail:CHAO@sc.edu

Y.J. Chao

School of Material Science and Engineering, Tianjin University,

Tianjin, China level interfacial mechanical model is presented to analyze interfacial bonding strengths of mesoscopic bundles and microscopic nanotubes. This study shows that properties of the multi-level interfaces are critical factors for determining fiber strength and toughness.

With the aid of the atomic force microscopy (AFM) indentation system, Dongchuan Su et al. studied the near-surface elastic modulus via force-penetration curves acquired during indentation. An analysis algorithm is proposed based on the secant modulus method to extract the true penetration depths from force-displacement curves in AFM indentation testing. The results show that the AFM indentation method is capable of producing high spatial resolution maps of the elastic modulus with small forces and shallow indentations.

MEMS sensors are typically fabricated out of materials that are mechanically sound at the microscale, but can be relatively poor electrical conductors. For this reason, areas of MEMS are coated with various thin metal films to provide electrical pathways. These films, however, adversely alter resonant properties of a device. The paper by Pryputniewicz reviews a theoretical analysis of the effect that thermoelastic internal friction has on the Q-factor of microscale resonators and shows that the internal friction relating to TED is a fundamental damping mechanism in determination of quality of high-Q resonators over a range of operating conditions.

As a method for measuring full-field out-of-plane displacement, projection moiré provides high quality results with simple test setup. B. Gulker et al. implemented a projection moiré system in low-velocity impact testing using an image processing program. Results from the projection moiré experiments agree reasonably well with those obtained from the commonly used load cell method. The technique is further applied to composites with various microstructures.

Y.R. Zhao et al. developed the electron grid fabricating technique by using a common scanning electron microscope (SEM). An error analysis for the multi-scanning grating was 
performed by a sampling moiré method. Optimal parameters for multi-scanning electron moiré method are given to produce fine nano-gratings. A high accelerating voltage or a short working distance yield better results generally. A cross-line grid with a frequency of 10,000 lines $/ \mathrm{mm}$ and a parallel grating with a frequency of 13,000 lines $/ \mathrm{mm}$ were successfully fabricated.

Feng Xu et al. investigated in situ the influence of second phase fine powders on solid state sintering by synchrotron radiation X-ray computed tomography (SR-CT). Typical sintering parameters, which control mechanical properties, were extracted from the experimental data, and the effects of the second phase powders were quantitatively analyzed. The results indicate that the $\mathrm{Si}_{3} \mathrm{~N}_{4}$ fine powders can enhance the sintering of the original $\mathrm{SiO}_{2}$ powders rather than hindering the process.

Jingpin Jiao et al. proposed a signal processing algorithm that can extract three nonlinear indicators related to amplitude attenuation, phase shift, and harmonics. The experiments demonstrated that the three nonlinear indicators were able to characterize the contact conditions of the interfaces studied, but their sensitivities to pressure differ for interfaces of different roughness and over different pressure ranges. The proposed nonlinear indicators can effectively provide information on the contact condition of the interfaces.

M.K. Eibatanouny et al. focused their work on the acoustic emission (AE) to monitor the cracking mechanisms leading to the failure of scaled FRP Reinforced concrete beam. Acoustic emission analyses were performed to classify crack types occurring at different points in the load history. The results of this study indicate that appropriate AE parameters can be used to discriminate between developing flexural and shear cracks irrespective of scale, and provide warning of impending failure irrespective of the failure mode (flexural and shear).

$\mathrm{H}$. Zhang et al. investigated the influence of MWCNTs (multi-walled carbon nanotubes) on the flexural property and the impact performance of GLAREs (Glass Reinforced
Aluminum Laminates) by the three-point bending and drop weight Dynatup impact testing, respectively. In comparison with pure epoxy bonded GLARE, the results show that the modified GLAREs can generally improve the impact resistance via diversifying energy absorbing patterns at nano level.

Junhong Zhou et al. proposed an experimental procedure called standard aiming target test to quantitatively evaluate the effect of vibrating insole on specific task of aiming during standing. Based on the calculation of multiscale entropies (MSE), they found that the vibrating insole can significantly improve the aiming performance and lead to increased entropy in the displacement fluctuations of aiming spot.

In sum, the papers in this special issue aim to report the latest progress in both technology/metrology and applications in related areas. The topics include characterization of the mechanical properties of material with in situ Raman spectroscopy, SR-CT, SEM and AFM, new technique for fabricating micro-moiré grating, development on theoretical models and the scale-independent theory for deformation analysis, and study on the acoustic emission metrology as well as applications to the problems of contact Interfaces and scaled FRP reinforced concrete structures. This special issue points to the great prospects for multi-scale metrologies and relevant applications, and should be of interest to researchers in mechanics, materials and other related fields. With the fast advancement in hardware in both small and large scale, the development of interdisciplinary research in crossing the boundaries of different lengthscales has progressed with a pace never seen before. It is hoped that this special issue can provide a framework and point to a lucid direction for colleagues to further advance the science and technology in multi-scale experimental mechanics.

The guest editors are very grateful to all the contributing authors for their significant contributions and great support. We also appreciate the reviewers for their invaluable assistance during the review process of all papers in this special issue. 